\title{
Statistical relevance of vorticity conservation in the Hamiltonian particle-mesh method is
}

\author{
Svetlana Dubinkina, Jason Frank* \\ CWI, P.O. Box 94079, 1090 GB Amsterdam, The Netherlands
}

\section{A R T I C L E I N F O}

\section{Article history:}

Received 7 August 2009

Received in revised form 8 December 2009

Accepted 8 December 2009

Available online 16 December 2009

\section{Keywords:}

Conservative discretizations

Statistical mechanics

Geometric numerical integration

Quasigeostrophic flow

Geophysical fluid dynamics

\begin{abstract}
A B S T R A C T
We conduct long-time simulations with a Hamiltonian particle-mesh method for ideal fluid flow, to determine the statistical mean vorticity field of the discretization. Lagrangian and Eulerian statistical models are proposed for the discrete dynamics, and these are compared against numerical experiments. The observed results are in excellent agreement with the theoretical models, as well as with the continuum statistical mechanical theory for ideal fluid flow developed by Ellis et al. (2002) [10]. In particular the results verify that the apparently trivial conservation of potential vorticity along particle paths within the HPM method significantly influences the mean state. As a side note, the numerical experiments show that a nonzero fourth moment of potential vorticity can influence the statistical mean.
\end{abstract}

(c) 2009 Elsevier Inc. All rights reserved.

\section{Introduction}

Computational applications in atmosphere and ocean science often involve the simulation of geophysical fluids on time intervals much longer than the Lyapunov e-folding time. In these instances the goal of simulation is the evaluation of statistical quantities such as averages and correlations. It is therefore important to investigate the accuracy of numerical discretizations in the context of statistical averages.

The Hamiltonian particle-mesh (HPM) method was originally proposed in the context of rotating shallow water flow in periodic geometry in [11] and extended to other physical settings in [6,13,7,28]. The fluid is discretized on a finite set of Lagrangian particles that transport the mass of the fluid and persist during the flow evolution. The HPM method is symplectic, and one can construct a continuum velocity field in which the discrete particle velocities are embedded for all time. The continuum velocity field satisfies a Kelvin circulation theorem, implying material conservation of potential vorticity and invariance of the infinite family of Casimir functionals, see [12].

For the case of ideal fluid flow in two dimensions, the HPM variant was described in [6]. We will apply the HPM method to the quasigeostrophic potential vorticity equation describing a 2D barotropic flow over topography. Here, it is the potential vorticity (PV), and not the mass, that is fixed on each particle and advected in a divergence-free velocity field. In this case we show that the particle motion may be embedded in an area-preserving flow on the fluid label space. Hence, an arbitrary function of PV may be integrated by quadrature over label space and is therefore conserved. On the other hand, this trivial conservation is apparently due to the fact that a value of PV is assigned to each particle once and for all, and does not imply any reduction of the number of degrees of freedom of the flow evolution in the sense of, say, a discrete energy conservation law.

\footnotetext{
The investigations were in part supported by the Research Council for Earth and Life Sciences (ALW) with financial aid from the Netherlands Organization for Scientific Research (NWO).

* Corresponding author.

E-mail addresses: dubinkin@cwi.nl (S. Dubinkina), jason@cwi.nl (J. Frank).
} 
In fact, the method makes use of a secondary (coarse-grain) vorticity field, defined on a uniform grid, and numerical simulations indicate that only energy and linear functionals of PV are conserved at this macroscopic scale.

Consequently, one may question to what degree the PV conservation of the HPM method is meaningful. Long time simulations of nonlinear dynamical systems are typically inaccurate in a point-wise sense and rather are carried out with the goal of sampling an equilibrium probability distribution over the phase space under an assumption of ergodicity. The relevant statistical equilibrium distribution is a function of the conservation laws that restrict the dynamics. Hence, if the PV conservation by HPM is truly trivial, the statistics should adhere to that of an energy-circulation equilibrium theory, whereas a meaningful PV conservation should lead to a richer statistical equilibrium. In this paper, we will show that the PV conservation of HPM significantly influences the statistics of simulation data obtained with the HPM method.

Sophisticated statistical equilibrium theories for ideal fluids are based on conservation of generalized enstrophiesthe integrals over the domain of arbitrary functions of PV-or, equivalently, on the area-preservation property of the velocity field. An equilibrium theory with microcanonical treatment of vorticity invariants was developed independently by Lynden-Bell [17], Robert [24], Robert and Sommeria [25], and Miller [20]. An alternative approach that treats vorticity invariants canonically was developed by Ellis et al. [10]. See also Chavanis [5] for a comparison of the two. Our numerical method has features in common with the model used to construct the latter continuum theory (specifically, a natural two-scale structure), and in this paper we derive analogous discrete statistical equilibrium models based on both Lagrangian (Section 4) and Eulerian (Section 5) fluid considerations and compare these models with simulations (Section 6).

We wish to emphasize here that the objective of the present paper is to show that the discrete statistics of the HPM method are in good agreement with predictions of the modern continuum theory. This implies that the construction of the HPM method respects the dynamical considerations that go into the theory, and that for the numerical experiments included, the discrete flow is sufficiently ergodic to observe convergence of the ensemble averages.

The quasigeostrophic potential vorticity (QG) equation [23,26,18] describes barotropic divergence-free flow over topography

$$
\frac{d}{d t} q(\boldsymbol{x}, t)=0, \quad \Delta \psi(\boldsymbol{x}, t)=q(\boldsymbol{x}, t)-h(\boldsymbol{x}),
$$

where $q$ is the potential vorticity (PV) field, $\psi$ is the stream function, and $h$ is the topography of the earth. The Laplacian operator is denoted by $\Delta$ and the material derivative by $\frac{d}{d t}=\frac{\partial}{\partial t}+\boldsymbol{u} \cdot \nabla$. Here, the divergence-free velocity field $\boldsymbol{u}$ is related to the stream function by $\boldsymbol{u}=\nabla^{\perp} \psi$, where $\nabla^{\perp}=\left(-\frac{\partial}{\partial y}, \frac{\partial}{\partial x}\right)^{T}$. In this paper, we consider the QG equation on a doubly periodic domain

$$
\boldsymbol{x}=(x, y) \in \mathcal{D} \equiv[0,2 \pi) \times[0,2 \pi)
$$

The QG model describes a Hamiltonian PDE with Lie-Poisson structure [26], implying the conservation of the total kinetic energy

$$
\mathcal{H}=-\frac{1}{2} \int_{\mathcal{D}} \psi \cdot(q-h) d \boldsymbol{x}
$$

as well as the infinite class of Casimir functionals

$$
\mathcal{C}[f]=\int_{\mathcal{D}} f(q) d \boldsymbol{x}
$$

for any function $f$ for which the integral exists. Of particular interest are the PV moments:

$$
\mathcal{C}_{r}=\int_{\mathcal{D}} q^{r} d \boldsymbol{x}, \quad r=1,2, \ldots,
$$

and especially the circulation $\mathcal{C}_{1}$ and enstrophy $\mathcal{C}_{2}$.

Preservation of the Casimir functionals follows from area-preservation under the divergence-free velocity field [21]: define a function $G(\sigma, t)$ denoting the measure of that part of the domain $\mathcal{D}$ for which the vorticity is less than $\sigma$ :

$$
G(\sigma, t)=\operatorname{meas}\{\boldsymbol{x} \in \mathcal{D} \mid q(\boldsymbol{x}, t)<\sigma\} .
$$

We note that due to the divergence-free advection of $q$, this function is independent of time $\frac{\partial G}{\partial t}=0$. Differentiating with respect to $\sigma$, the function

$$
g(\sigma)=\frac{\partial G}{\partial \sigma}
$$

is preserved. For the case of a piecewise uniform PV field, $q(\boldsymbol{x}, t) \in\left\{\sigma_{1}, \ldots, \sigma_{L}\right\}$, the quantity $g_{\ell}=G\left(\sigma_{\ell+1}\right)-G\left(\sigma_{\ell}\right)$ is the measure of the vorticity level set $\sigma_{\ell}$. 


\section{Review of continuum statistical equilibrium theories}

Given a (spatially) discrete approximation $\boldsymbol{q}(t)$ to the solution $q(\boldsymbol{x}, t)$ of $(1)$, obtained from a numerical simulation, one would like to analyze the accuracy of computed averages of functions of the solution. For example, the long time average of a function $F(\boldsymbol{q}(t))$ of the PV field is denoted

$$
\bar{F}=\lim _{T \rightarrow \infty} \frac{1}{T} \int_{t_{0}}^{t_{0}+T} F(\boldsymbol{q}(t)) d t .
$$

If the discrete dynamics is ergodic with respect to a unique invariant measure $p(\boldsymbol{q})$ on the phase space, then the long time average is equivalent to the ensemble average with respect to $p$,

$$
\langle F(\boldsymbol{q})\rangle=\int F(\boldsymbol{q}) p(\boldsymbol{q}) d \boldsymbol{q},
$$

where the integral is over the (function) space of PV fields, and it suffices to derive the invariant measure associated with the numerical method, and analyze this with respect to what is known about the continuum model.

In an effort to characterize the long time mean behavior of ideal fluids and explain their tendency to organize into largescale coherent structures, a number of authors have applied ideas from statistical mechanics. The pioneering work was that of Onsager [22], which addressed the statistical mechanics of a finite point vortex model. He observed that for a bounded domain, the available phase space must eventually decrease as a function of increasing system energy, leading to negative temperature regimes in the microcanonical statistical ensemble. He also predicted that in a heterogeneous system of likesigned and oppositely-signed vortices of varying strengths, the large vortices would tend to cluster so as to achieve maximum disorder with minimum degrees of freedom, like-signed vortices at negative temperatures and vice-versa. These predictions were confirmed numerically in [3].

Statistical mechanics theories based on a Fourier-spectral truncation of the Euler equations were proposed by Kraichnan [15], Salmon et al. [27], and Carnevale and Frederiksen [4]. The spectral truncation preserves circulation $\mathcal{C}_{1}$ and the quadratic functions $\mathcal{E}$ and $\mathcal{C}_{2}$ only. Consequently, a treatment based on constrained maximum entropy (for an introduction, see [18]) yields Gibbs-like distributions $p(q)$ over the vorticity field, with Gaussian distribution of local vorticity fluctuations. The energy-enstrophy theory predicts a linear relation between ensemble average stream function and potential vorticity:

$$
\langle q(x)\rangle=\mu\langle\psi(x)\rangle
$$

for a scalar $\mu$ depending on the observed energy and enstrophy, where the ensemble average is defined with respect to integration over an appropriate function space in this case.

Given that it is not just the enstrophy $\mathcal{C}_{2}$ but any functional $\mathcal{C}[f]$ that is invariant under the continuum flow of (1), it is natural to ask what effect the more general conservation laws have on statistics. Abramov and Majda [1] investigated the statistical significance of the higher PV moments $\mathcal{C}_{r}, r>2$, numerically using the Poisson discretization of Zeitlin [29] and McLachlan [19], which conserves $M$ Casimirs of an $M \times M$-mode truncation. Computing the long time-averaged PV and stream function fields, they observed increasing discrepancy relative to the linear mean field theory (7), as a function of increasing skewness $\mathcal{C}_{3}$ of the initial condition, thus proving the statistical relevance of this quantity.

Statistical equilibrium theories incorporating the full family of Casimirs implied by the preservation of area (5) were independently proposed by Lynden-Bell [17] in the context of astrophysics, and Miller [20], Miller et al. [21], Robert [24], and Robert and Sommeria [25]. These original theories used a microcanonical treatment of the Casimirs $\mathcal{C}[f]$. That is, the equilibrium distribution is derived by minimizing entropy under constraints of energy and the entire family of Casimirs. More recently, Ellis et al. [10] proposed an alternative theory featuring canonical treatment of the Casimirs, in which the point statistics of PV is described by a prior distribution. In all of these papers, a coarse-grain potential vorticity field is described by a probability density over the class of fine-grain PV distributions at each point in the domain.

In [9], we analyzed energy and enstrophy conserving finite difference methods for the QG model under topographic forcing, and observed that the discrete time-averaged mean fields $\overline{\boldsymbol{q}}$ and $\bar{\psi}$ obtained depend heavily on the conservation properties of the discretizations used. For a discretization that conserves energy only, the predicted mean field is uniformly zero velocity $\langle\Psi\rangle=0$. Given that the only dynamically conserved quantities of the HPM method are energy and total circulation (see below), any departure from the trivial mean field is an indication of the statistical relevance of the other conserved quantities, namely the area measure (5).

\section{Hamiltonian particle-mesh method}

The Hamiltonian particle-mesh (HPM) method is a numerical discretization of inviscid fluid dynamics that retains Hamiltonian structure. The method makes use of a Lagrangian fluid description, to advect fluid particles while conserving energy, and an Eulerian grid for evaluating derivatives using finite differences. The method was adapted for $2 \mathrm{D}$ incompressible flow in [6]. 


\subsection{HPM description}

The PV field is discretized by introducing a set of $K$ discrete particles with fixed potential vorticity $Q_{k}, k=1, \ldots, K$. The particles have time-dependent position $\boldsymbol{X}_{k}(t) \in \mathcal{D}$, and are advected in a divergence-free velocity field according to

$$
\frac{d}{d t} \boldsymbol{X}_{k}=\left.\nabla^{\perp} \Psi(\boldsymbol{x}, t)\right|_{\boldsymbol{x}=\boldsymbol{X}_{k}(t)},
$$

where the stream function $\Psi$ is described below.

We also make use of a uniform $M \times M$ grid on $\mathcal{D}$, with grid spacing $\Delta x=\Delta y=2 \pi / M$, and denote gridpoints by $\boldsymbol{x}_{i}$. Given a discrete stream function $\Psi_{i}(t)$ on the grid, we construct a continuous field via

$$
\Psi(\boldsymbol{x}, t)=\sum_{i} \Psi_{i}(t) \phi_{i}(\boldsymbol{x})
$$

where $\phi_{i}(\boldsymbol{x})=\phi\left(\frac{\boldsymbol{x}-\boldsymbol{x}_{i}}{\Delta x}\right)$ is a compactly supported basis function satisfying symmetry, normalization and partition of unity properties, respectively:

$$
\phi(\boldsymbol{x})=\phi(-\boldsymbol{x}), \quad \int_{\mathcal{D}} \phi(\boldsymbol{x}) d \boldsymbol{x}=1, \quad \sum_{i} \phi_{i}(\boldsymbol{x})=1, \quad \forall \boldsymbol{x} \in \mathcal{D} .
$$

In our implementation we use the tensor product of normalized cubic B-splines $\phi(\boldsymbol{x})=\phi_{0}(x) \phi_{0}(y)$, where

$$
\phi_{0}(r)= \begin{cases}\frac{2}{3}-|r|^{2}+\frac{1}{2}|r|^{3}, & |r| \leqslant 1 \\ \frac{1}{6}(2-|r|)^{3}, & 1<|r| \leqslant 2 \\ 0, & \text { otherwise. }\end{cases}
$$

The discrete stream function $\Psi_{i}(t)$ is obtained by solving a Poisson equation on the grid. Given a discrete grid-based PV field $q_{i}(t)$ we solve

$$
\sum_{j} \Delta_{i j} \Psi_{j}=q_{i}-h_{i}
$$

where $h_{i}=h\left(\boldsymbol{x}_{i}\right)$ is the topography function sampled at gridpoints and $\Delta_{i j}$ is an appropriate discretization of the Laplacian. In our implementation, we use a spectral approximation and FFTs, but a finite difference formula may be sufficient.

Finally, the PV field on the grid is approximated from the particles using the relation

$$
q_{i}(t)=\sum_{k} Q_{k} \phi_{i}\left(\boldsymbol{X}_{k}(t)\right)
$$

In [12] it is shown that the above formula samples the exact solution of a continuity equation of the form $q_{t}+\nabla \cdot(q \hat{\boldsymbol{u}})=0$ with density function $q(\boldsymbol{x}, t)=\sum_{k} Q_{k} \phi\left(\boldsymbol{x}-\boldsymbol{X}_{k}\right)$ and auxiliary velocity field $\hat{\boldsymbol{u}}(\boldsymbol{x}, t)$ appropriately defined. In the present case, although the particle velocity field is given by $\nabla^{\perp} \Psi(\boldsymbol{x}, t)$ and is therefore divergence-free, this will only hold in an approximate sense for the auxiliary velocity field $\hat{\boldsymbol{u}}(\boldsymbol{x}, t)$.

In the present context of vortex dynamics, the HPM method is related to the classical point vortex flow (see [8] and references therein). The singular point vortices have been regularized by convolution with the basis functions $\phi$. The Eulerian grid reduces the complexity of vortex-vortex interactions from $\mathcal{O}\left(K^{2}\right)$ to $\mathcal{O}(K \ln K)$ (using FFT). The construction of the method preserves the Hamiltonian structure of the point vortex flow. However, as noted in the introduction, the HPM method was originally in the setting of compressible flow and is in this sense applicable to more general fluids than the point vortex model.

\subsection{Properties of the discretization}

By construction, the numerical method described above defines a Hamiltonian system. The Hamiltonian is

$$
H(\boldsymbol{X})=-\frac{1}{2} \sum_{i} \Psi_{i}\left(q_{i}-h_{i}\right) \Delta x^{2}=-\frac{1}{2} \sum_{i, j}\left[\left(\sum_{k} Q_{k} \phi_{i}\left(\boldsymbol{X}_{k}\right)\right)-h_{i}\right]\left(\Delta^{-1}\right)_{i, j}\left[\left(\sum_{\ell} Q_{\ell} \phi_{j}\left(\boldsymbol{X}_{\ell}\right)\right)-h_{j}\right] \Delta x^{2} .
$$

Introducing phase space coordinates $\boldsymbol{X}=\left(X_{1}, \ldots, X_{K}, Y_{1}, \ldots, Y_{K}\right)^{T}$ and symplectic two-form structure matrix

$$
B=\left[\begin{array}{cc}
0 & -\operatorname{diag} \boldsymbol{Q} \\
\operatorname{diag} \boldsymbol{Q} & 0
\end{array}\right]
$$

the equations of particle motion are described by

$$
B \frac{d \boldsymbol{X}}{d t}=\nabla H(\boldsymbol{X})
$$


The Hamiltonian is a first integral of the dynamics and approximates the total kinetic energy. Additionally, the phase flow is symplectic and consequently volume-preserving on $\mathbb{R}^{2 K}$.

To integrate the numerical discretization in time we use the implicit midpoint rule:

$$
B \frac{\boldsymbol{X}^{n+1}-\boldsymbol{X}^{n}}{\Delta t}=\nabla H\left(\frac{\boldsymbol{X}^{n+1}+\boldsymbol{X}^{n}}{2}\right) .
$$

The numerical map is symplectic for this problem, implying that volume is preserved in the $2 K$-dimensional phase space of particle positions. Also the energy is well-preserved, with fluctuations bounded by a term of $\mathcal{O}\left(\Delta t^{2}\right)$ for long times, consistent with theory reported in $[14,16]$.

Since the particle PV values $Q_{k}, k=1, \ldots, K$ are fixed for the duration of the computation, PV is conserved along particle paths for any motion of the particles. However, since the $Q_{k}$ play the role of parameters in the specification of HPM, their conservation does not imply a reduction in degrees of freedom of the dynamics in the way exact conservation of $H$ does. On the other hand, the motion of the particles is not arbitrary, but area-preserving in the sense described next. The combination of material conservation of PV in an area-preserving flow is the essential feature of the fine scale motion of ideal fluids that enters into the modern statistical mechanics theories.

Given an arbitrary continuous motion of the particles $\boldsymbol{X}_{k}(t)$, Eqs. (11), (10) and (8) define a continuum approximate stream function $\Psi(\boldsymbol{x}, t)$, with velocity field

$$
\boldsymbol{U}(\boldsymbol{x}, t)=\nabla^{\perp} \Psi(\boldsymbol{x}, t) .
$$

Let us define label coordinates $\boldsymbol{a}=(a, b)$ on $\mathcal{D}$ and the Lagrangian flow $\chi(\boldsymbol{a}, t): \mathcal{D} \times \mathbb{R} \rightarrow \mathcal{D}$ induced by $\Psi(\boldsymbol{x}, t)$ :

$$
\frac{\partial}{\partial t} \boldsymbol{\chi}(\boldsymbol{a}, t)=\boldsymbol{U}(\boldsymbol{\chi}(\boldsymbol{a}, t), t) .
$$

Since $\nabla \cdot \boldsymbol{U} \equiv 0$, the Lagrangian flow $\chi$ is area-preserving on $\mathcal{D}$. That is,

$$
\left|\operatorname{det} \frac{\partial \boldsymbol{\chi}}{\partial \boldsymbol{a}}\right| \equiv 1 \text {. }
$$

This property is retained under temporal semi-discretization with the implicit midpoint rule. That is, the mapping $\chi^{n}(\boldsymbol{a}) \mapsto \chi^{n+1}(\boldsymbol{a})$ is area-preserving.

On the other hand, for the numerical method, the particle motion $\boldsymbol{X}_{k}(t)$ is just given by

$$
\boldsymbol{X}_{k}(t)=\chi\left(\boldsymbol{X}_{k}(0), t\right),
$$

i.e. the particle motion is embedded in its own Lagrangian flow. Therefore, the discrete particle motion is area-preserving in the sense that it can be embedded in an area-preserving flow.

Typically, we initialize the particles on a uniform grid $^{1}$ with spacing $\Delta a=\Delta x / \kappa$ for some positive integer $\kappa$. Let $\boldsymbol{a}_{k} \in \mathcal{D}$ denote the initial position of the $k$ th particle. Let $A_{k}$ denote the set of labels in the grid cell centered at $\boldsymbol{a}_{k}$ :

$$
A_{k}=\left\{\boldsymbol{a} \in \mathcal{D}:\left|\boldsymbol{a}-\boldsymbol{a}_{k}\right|_{\infty}<\frac{\Delta a}{2}\right\} .
$$

Then $\mathcal{D}=\cup_{k} \overline{A_{k}}$. Define a piecewise constant initial vorticity field through

$$
Q_{0}(\boldsymbol{a})=\sum_{k} Q_{k} \mathbf{1}_{k}(\boldsymbol{a})
$$

where $\mathbf{1}_{k}$ is the characteristic function on $A_{k}$. This vorticity field is transported by the flow $\chi(\boldsymbol{a}, t)$ via

$$
Q(\chi(\boldsymbol{a}, t), t)=Q_{0}(\boldsymbol{a}) .
$$

Given any function $f(Q)$, we have

$$
\begin{aligned}
\int_{\mathcal{D}} f(Q(\boldsymbol{x}, t)) d \boldsymbol{x} & =\int_{\mathcal{D}} f(Q(\chi(\boldsymbol{a}, t), t))\left|\operatorname{det} \frac{\partial \boldsymbol{\chi}}{\partial \boldsymbol{a}}\right| \operatorname{da}=\int_{\mathcal{D}} f\left(Q_{0}(\boldsymbol{a})\right)\left|\operatorname{det} \frac{\partial \boldsymbol{\chi}}{\partial \boldsymbol{a}}\right| \operatorname{da}=\sum_{k} f\left(Q_{k}\right) \int_{A_{k}}\left|\operatorname{det} \frac{\partial \boldsymbol{\chi}}{\partial \boldsymbol{a}}\right| \operatorname{da}=\sum_{k} f\left(Q_{k}\right)\left|A_{k}\right| \\
& =\Delta a^{2} \sum_{k} f\left(Q_{k}\right),
\end{aligned}
$$

which is constant. In particular, the area associated with any particular level set of PV is conserved.

In this sense we see that the fine scale particle flow trivially conserves all Casimirs, and in particular the polynomials functions

$$
C_{r}=\Delta a^{2} \sum_{k} Q_{k}^{r}, \quad r=1,2, \ldots
$$

\footnotetext{
${ }^{1}$ For an arbitrary initial particle configuration, the subsequent quadrature could be carried out on the Voronoi cells.
} 
However, this property does not transfer to the gridded PV field $\boldsymbol{q}$. That is, the grid-based analogs

$$
\widehat{C}_{r}=\Delta x^{2} \sum_{i} q_{i}^{r}, \quad r=1,2, \ldots,
$$

are not conserved in general. The sole exception is the total circulation $\widehat{C}_{1}$ for which we have, using the third property of (9),

$$
\widehat{C}_{1}=\sum_{i} q_{i} \Delta x^{2}=\sum_{i} \sum_{k} Q_{k} \phi_{i}\left(X_{k}\right) \kappa^{2} \Delta a^{2}=\sum_{k} Q_{k} \kappa^{2} \Delta a^{2},
$$

which is independent of time. For arbitrary nonlinear $f(q)$, one would not expect the quantity $\sum_{i} f\left(q_{i}\right) \Delta x^{2}$ to be invariant in general.

In Fig. 1, we plot the relative drift

$$
\varepsilon_{\mathrm{rel}}[H](t)=\left|\frac{H(t)-H(0)}{H(0)}\right|
$$

in the quantities $H$ and $\widehat{C}_{r}, r=2, \ldots, 4$ as a functions of time during a typical simulation (the experiment described in Section 6.1.2, for the case $\gamma=0, \delta=90$ ). The circulation is preserved to machine precision and is not shown. The energy oscillations are bounded by

$$
\varepsilon_{\mathrm{rel}}[H](t)<2.1 \times 10^{-4},
$$

and the bound decreases quadratically with stepsize. The higher order vorticity moments are not preserved, and encounter relative drifts

$$
\max _{t} \varepsilon_{\mathrm{rel}}\left[\widehat{C}_{2}\right](t)=0.74, \quad \max _{t} \varepsilon_{\mathrm{rel}}\left[\widehat{C}_{3}\right](t)=16.9, \quad \max _{t} \varepsilon_{\mathrm{rel}}\left[\widehat{C}_{4}\right](t)=16.5
$$

Clearly, these are not conserved.

In some cases, it is useful to consider the bulk motion of the fluid to be prescribed by a time-dependent stream function $\Psi(\boldsymbol{x}, t)$, and consider the motion of a typical particle embedded in the flow. The motion of such a particle satisfies a nonautonomous Hamiltonian system.

This point of view and its coupling to the dynamics is studied in [2]. The parcel Hamiltonian becomes

$$
\widetilde{\mathcal{H}}=\int q_{0}(\boldsymbol{a}) \Psi(\boldsymbol{\chi}(\boldsymbol{a}, t), t) d \boldsymbol{a},
$$

and the dynamics on label space (13) satisfy

$$
q_{0}(\boldsymbol{a}) \frac{d}{d t} \boldsymbol{\chi}(\boldsymbol{a}, t)=J \frac{\delta \widetilde{\mathcal{H}}}{\delta \boldsymbol{\chi}}=q_{0}(\boldsymbol{a}) \nabla^{\perp} \Psi(\boldsymbol{\chi}(\boldsymbol{a}, t), t), \quad \forall \boldsymbol{a} .
$$

where $J=\left[\begin{array}{cc}0 & I \\ -I & 0\end{array}\right]$.

Similarly, for the HPM discretization,

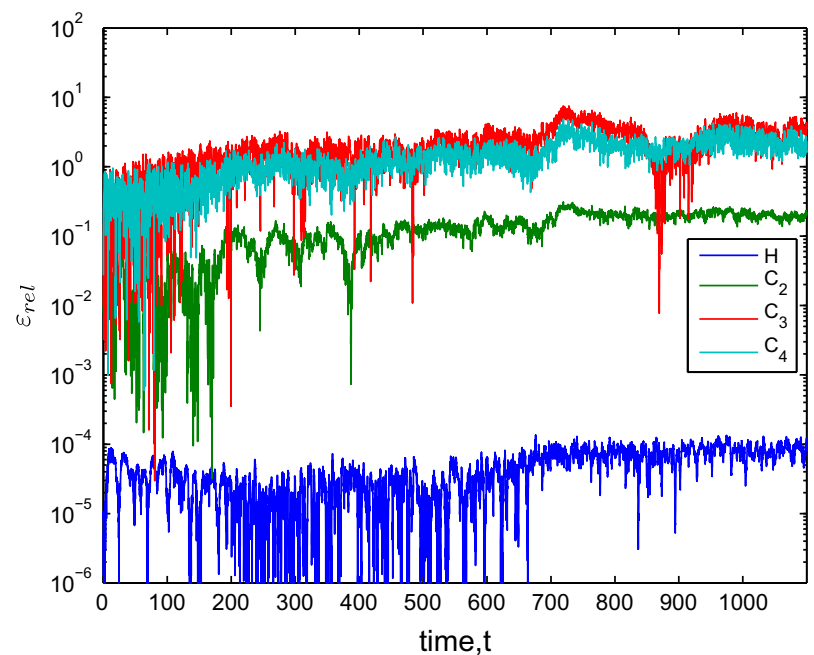

Fig. 1. Relative change in energy $H$ and higher vorticity moments $\widehat{C}_{2}, \widehat{C}_{3}, \widehat{C}_{4}$ during the simulation described in Section 6.1 .2 , for the case $\gamma=0, \delta=90$. 


$$
Q_{k} \frac{\partial}{\partial t} \boldsymbol{X}_{k}=J \nabla_{\boldsymbol{X}_{k}(t)} \widetilde{H}=Q_{k} \nabla^{\perp} \Psi\left(\boldsymbol{X}_{k}(t), t\right),
$$

where

$$
\widetilde{H}=\sum_{k} Q_{k} \Psi\left(\boldsymbol{X}_{k}(t), t\right)
$$

\section{A Lagrangian statistical model based on canonical particle distributions}

Due to its Lagrangian nature, the HPM method has similarities with the point vortex method whose statistical mechanics was considered by Onsager [22]. The particle motion can be considered a regularized point vortex method. The phase space of the HPM method is bounded: it is simply $\mathcal{D}^{K}$. In contrast to the point vortex method, the range of energy for the HPM method is also bounded (for finite $Q_{k}, k=1, \ldots, K$ ). If one considers possible configurations for a given energy, as the energy level becomes large enough the available phase space eventually starts to decrease. In other words, the HPM method supports a negative temperature regime.

In this section, we construct a statistical equilibrium theory in the natural phase space of HPM particle positions $\boldsymbol{X}_{k} \in \mathcal{D}$. However, in some cases it may be preferable to directly consider the statistics of the coarse-grain vorticity field $\mathbf{q}$ on the grid (11), which allows comparison with the existing equilibrium field theories. In the next section, we present an Eulerian approximate statistical model from this point of view. To distinguish the two, we refer to the theory in this section as the Lagrangian statistical mechanical model.

Let us consider the statistics of a single distinguished particle in contact with the reservoir formed by all other particles. Recall that the motion of such a particle obeys a nonautonomous Hamiltonian system (17) with Hamiltonian (18). The energy contribution of particle $k$ is $Q_{k} \Psi\left(\boldsymbol{X}_{k}, t\right)$. We expand the stream function about the ensemble mean field

$$
\Psi(\boldsymbol{x}, t)=\langle\Psi(\boldsymbol{x})\rangle+\delta \Psi(\boldsymbol{x}, t) .
$$

Neglecting the long time effects of the perturbation part $\delta \Psi$, we obtain the canonical distribution for a distinguished particle

$$
\rho_{k}(\boldsymbol{x})=\frac{1}{\zeta_{k}} e^{-\beta\langle\Psi(\boldsymbol{x})\rangle Q_{k}}, \quad \zeta_{k}=\int_{\boldsymbol{x} \in \mathcal{D}} e^{-\beta\langle\Psi(\boldsymbol{x})\rangle Q_{k}} d \boldsymbol{x} .
$$

Fig. 2 compares typical functions $\rho_{k}(x)$ with histograms of position data for two arbitrarily chosen particles with $Q_{k^{+}}=1.098$ and $Q_{k^{-}}=-2.165$ obtained from HPM simulations with normally distributed $\left\{Q_{k}\right\}$. We observe good agreement. Due to the choice of topography in Section 6 and normally distributed $Q_{k}$, the distributions $\rho_{k}$ are uniform in the $y$ direction.

The one-particle canonical statistics can be used to construct a mean field theory. For particle $k$ the one-particle statistics is (19). This quantity gives the probability that $\boldsymbol{X}_{k}$ is near $\boldsymbol{x} \in \mathcal{D}$. Next consider coordinates $\boldsymbol{\Xi}=\left(\boldsymbol{\xi}_{1}, \boldsymbol{\xi}_{2}, \ldots, \boldsymbol{\xi}_{K}\right) \in \mathcal{D}^{K}$ on the particle phase space, and the product distribution

$$
\rho(\boldsymbol{\Xi})=\prod_{k} \rho_{k}\left(\boldsymbol{\xi}_{k}\right)
$$

which governs the probability of particle configurations under the modelling assumption that the particle positions are independent.

To each $\boldsymbol{\Xi} \in \mathcal{D}^{K}$, is an associated grid-based PV field $\boldsymbol{q}(\boldsymbol{\Xi})$ with
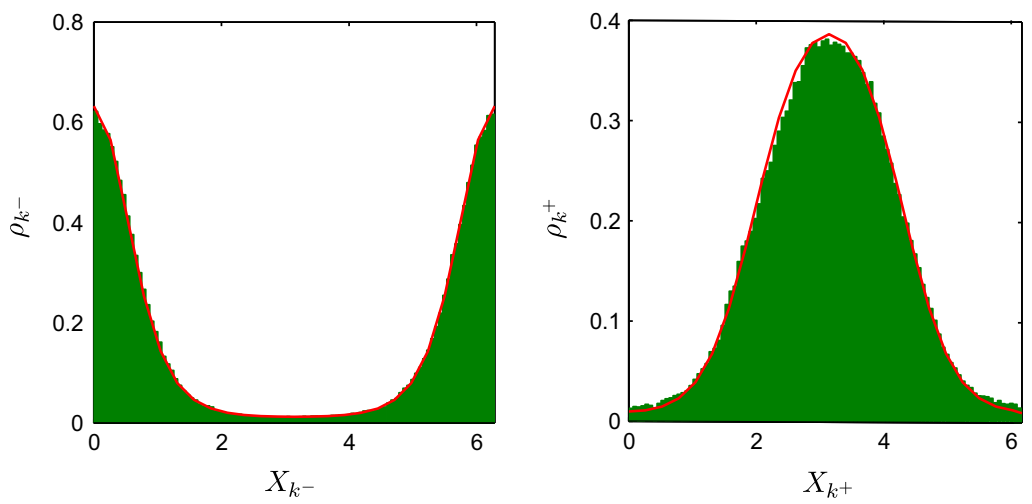

Fig. 2. Histograms of $x$-component of position for two distinct particles, compared with the predicted canonical distribution (in red). (For interpretation of the references in color in this figure legend, the reader is referred to the web version of this article.) 


$$
q_{i}(\boldsymbol{\Xi})=\sum_{k} Q_{k} \phi_{i}\left(\xi_{k}\right)
$$

The ensemble average PV field is

$$
\left\langle q_{i}\right\rangle=\int q_{i}(\boldsymbol{\Xi}) \rho(\boldsymbol{\Xi}) d \boldsymbol{\Xi},
$$

which can be simplified as follows

$$
\left\langle q_{i}\right\rangle=\int_{\mathcal{D}^{k}} \sum_{k} Q_{k} \phi_{i}\left(\xi_{k}\right) \prod_{\ell} \rho_{\ell}\left(\xi_{\ell}\right) d \xi_{1} \cdots d \xi_{K}=\sum_{k} Q_{k} \int_{\mathcal{D}} \phi_{i}(\boldsymbol{x}) \rho_{k}(\boldsymbol{x}) d \boldsymbol{x}=\sum_{k} Q_{k} \frac{\int_{\mathcal{D}} \phi_{i}(\boldsymbol{x}) e^{-\beta\langle\psi(\boldsymbol{x})\rangle Q_{k}} d \boldsymbol{x}}{\int_{\mathcal{D}} e^{-\beta\langle\psi(\boldsymbol{x})\rangle Q_{k}} d \boldsymbol{x}}=\sum_{k} Q_{k}\left\langle\phi_{i}\right\rangle_{k}
$$

where $\langle\cdot\rangle_{k}$ is the ensemble average in the measure (19).

If we consider a piecewise constant vorticity distribution with $K_{\ell}$ particles with PV $\sigma_{\ell}$, the relations above can be expressed as

$$
\left\langle q_{i}\right\rangle=\sum_{\ell} \sigma_{\ell} K_{\ell} \frac{\int_{\mathcal{D}} \phi_{i}(\boldsymbol{x}) e^{-\beta\langle\psi(\boldsymbol{x})\rangle \sigma_{\ell}} d \boldsymbol{x}}{\int_{\mathcal{D}} e^{-\beta\langle\psi(\boldsymbol{x})\rangle \sigma_{\ell}} d \boldsymbol{x}}=\sum_{\ell} \sigma_{\ell} p_{i, \ell},
$$

where

$$
p_{i, \ell}=K_{\ell}\left\langle\phi_{i}\right\rangle_{\ell} .
$$

This quantity is the proportion of time that a particle with PV $\sigma_{\ell}$ spends within the support of grid point $\boldsymbol{x}_{i}$, weighted by the kernel function, times the number of such particles.

To compute the mean state, we approximate the integral in (20) by quadrature at the grid points

$$
\left\langle q_{i}\right\rangle \approx \sum_{\ell} \sigma_{\ell} K_{\ell} \frac{\sum_{j} \phi_{i}\left(\boldsymbol{x}_{j}\right) e^{-\beta\left\langle\psi_{j}\right\rangle \sigma_{\ell}}}{\sum_{j} e^{-\beta\left\langle\psi_{j}\right\rangle \sigma_{\ell}}}
$$

This relation is solved together with

$$
\Delta_{i j}\left\langle\psi_{j}\right\rangle=\left\langle q_{i}\right\rangle-h_{i}
$$

and the constraint relation

$$
H(\langle\mathbf{q}\rangle)=H_{0},
$$

which specifies the value of $\beta$.

Our Lagrangian statistical theory for the HPM method is analogous to the canonical theory of Ellis et al. [10], inasmuch as the energy is treated microcanonically through the specification of $\beta$, and the fine-scale vorticity conservation is treated canonically.

\section{Eulerian statistical model for HPM}

The continuum statistical mechanics theories of Miller [20], Miller et al. [21], Robert [24], and Robert and Sommeria [25] can be constructed using a two-level discretization of the continuum vorticity field. The microscopic configuration space consists of permutations of a piecewise uniform vorticity field, assuming constant values on each cell of a fine mesh. The macroscopic vorticity field is the local average of the microscopic field on an embedding coarse mesh. The continuum theory is obtained by first letting the fine mesh size tend to zero for fixed coarse grid mesh size, and subsequently taking the continuum limit of the coarse mesh.

A similar approach-neglecting the continuum limits-can be used to construct a discrete statistical model for the HPM method. Keeping in mind the interpolating continuum flow (15), we define $p_{i}^{\ell}$ to be the probability of observing $Q\left(\boldsymbol{x}_{i}, t\right)=\sigma_{\ell}$ near grid point $\boldsymbol{x}_{i}$. Then $p_{i}^{\ell}$ has the properties

$$
\sum_{i} p_{i}^{\ell} \Delta x^{2}=g_{\ell}, \quad \sum_{\ell} p_{i}^{\ell}=1
$$

The first of these says the area of PV level sets is conserved and is the discrete analog of (6). The second says the flow is incompressible.

It is natural to associate $p_{i}^{\ell}$ with the characteristic functions at the grid points, smoothed by the HPM basis functions $\phi$. Denote by $\mathbb{K}_{\ell}$ the index set of particles with vorticity level $\sigma_{\ell}(\ell=1, \ldots, L$, for some $L \leqslant K)$ :

$$
\mathbb{K}_{\ell}=\left\{1 \leqslant k \leqslant K: Q_{k}=\sigma_{\ell}\right\} .
$$

Define the function 


$$
\varphi_{i}^{\ell}=\sum_{k \in \mathbb{K}_{\ell}} \phi_{i}\left(\boldsymbol{X}_{k}\right) \frac{1}{\kappa^{2}} .
$$

If the particles are initialized on a uniform grid of spacing $\Delta a=\Delta x / \kappa$, for $\kappa \geqslant 1$ an integer, then $\varphi_{i}^{\ell}$ has the required properties (22). To construct a Miller/Robert theory for the HPM method, we would initialize the particles on such a uniform grid, and consider permutations of the $Q_{k}$ as an approximation of the configuration space.

The motion of particles in the HPM method conserves energy, as pointed out in the Section 3. It is therefore necessary to further restrict the sample space to those permutations of PV that preserve the initial energy to within some tolerance. Define the coarse-grain mean potential vorticity by

$$
\left\langle q_{i}\right\rangle=\sum_{\ell} p_{i}^{\ell} \sigma_{\ell}
$$

the coarse-grain mean stream function by

$$
\Delta\langle\boldsymbol{\Psi}\rangle=\langle\boldsymbol{q}\rangle-\boldsymbol{h},
$$

and the energy of the mean field by

$$
H(\langle\mathbf{q}\rangle)=-\frac{1}{2}\langle\boldsymbol{\Psi}\rangle^{T}(\langle\boldsymbol{q}\rangle-\boldsymbol{h}) \Delta x^{2} .
$$

Substituting $\varphi_{i}^{\ell}$ for $p_{i}^{\ell}$ in (23), the above definitions are consistent with the (coarse-grain) grid quantities $\boldsymbol{q}$, $\Psi$, and $H$ given in (11), (10), and (12), respectively.

A microcanonical statistical model analogous to the Miller/Robert approach proceeds at this point by introducing the Shannon information entropy

$$
S=-\sum_{i, \ell} p_{i}^{\ell} \ln p_{i}^{\ell}
$$

and maximizing this function with respect to $p_{i}^{\ell}$ subject to constraints of observed values of energy, $H(\langle\boldsymbol{q}\rangle)=H_{0}$, and the conditions (22).

Instead we take here the alternative approach proposed by Ellis et al. [10] (henceforth, the EHT theory), which assumes a canonical ensemble with respect to the higher order Casimirs, as determined by a prior distribution over point-wise vorticity, in combination with a microcanonical distribution with respect to $\mathcal{H}$ and $\mathcal{C}_{1}$. This is consistent with observations of inviscid fluids, where $\mathcal{H}$ and $\mathcal{C}_{1}$ depend only on the large-scale vorticity, whereas the $\mathcal{C}_{r}, r>1$ depend on the fine scale detailed vorticity and the length scale of averaging. To that end we drop the requirement that $p_{i}^{\ell}$ satisfy the first condition of (22).

Given a set of particles initialized on a uniform grid with PV values $Q_{k}, k=1, \ldots, K$, we consider the associated piecewise constant continuum vorticity field as described in (14) and (15). To each vorticity level set $\sigma_{\ell}, \ell=1, \ldots, L$ we associate the fractional area

$$
\Pi_{\ell}=\frac{K_{\ell} \Delta a^{2}}{|\mathcal{D}|},
$$

where $K_{\ell}$ is the number of particles with vorticity $\sigma_{\ell}$ and $|\mathcal{D}|$ is the total area of $\mathcal{D}$. Note that $\sum_{\ell} \Pi_{\ell}=1$. We take $\Pi_{\ell}$ to be the prior distribution on PV. Given no other information about the flow, $\Pi_{\ell}$ is the probability of observing PV value $\sigma_{\ell}$ at an arbitrarily chosen point in $\mathcal{D}$. The probability is uniform in space.

To determine the probability distribution $p_{i}^{\ell}$ we maximize the relative entropy

$$
S(p, \Pi)=-\sum_{i, \ell} p_{i}^{\ell} \ln \frac{p_{i}^{\ell}}{\Pi_{\ell}}
$$

Given no other information about the system, we can maximize this entropy as a function of $p_{i}^{\ell}$ to find

$$
p_{i}^{\ell}=\Pi_{\ell},
$$

which is the prior distribution at each point on the grid, confirming the earlier statement.

Instead we wish to maximize (26) subject to microcanonical constraints on the energy

$$
E=H(\langle\boldsymbol{q}\rangle)-H_{0}=0,
$$

and the circulation

$$
\Gamma=\widehat{C}_{1}(\langle\boldsymbol{q}\rangle)-\widehat{C}_{1}(\mathbf{q}(0))=0,
$$

as well as the normalization constraints

$$
N_{i}=\sum_{\ell} p_{i}^{\ell}-1=0, \quad \forall i .
$$


Introducing Lagrange multipliers $\beta, \alpha$, and $\lambda_{i}$, respectively, for these constraints, we solve

$$
\frac{\partial S}{\partial p_{i}^{\ell}}+\beta \frac{\partial E}{\partial p_{i}^{\ell}}+\alpha \frac{\partial \Gamma}{\partial p_{i}^{\ell}}+\sum_{j} \lambda_{j} \frac{\partial N_{j}}{\partial p_{i}^{\ell}}=0 .
$$

The respective derivatives are

$$
\begin{aligned}
\frac{\partial S}{\partial p_{i}^{\ell}} & =-\left(\ln \frac{p_{i}^{\ell}}{\Pi_{\ell}}+1\right) \\
\frac{\partial E}{\partial p_{i}^{\ell}} & =\sum_{j} \frac{\partial H}{\partial\left\langle q_{j}\right\rangle} \frac{\partial\left\langle q_{j}\right\rangle}{\partial p_{i}^{\ell}}=-\sum_{j}\left\langle\Psi_{j}\right\rangle \sigma_{\ell} \delta_{i j} \Delta x^{2}=-\left\langle\Psi_{i}\right\rangle \sigma_{\ell} \Delta x^{2} \\
\frac{\partial \Gamma}{\partial p_{i}^{\ell}} & =\sigma_{\ell} \Delta x^{2} \\
\frac{\partial N_{j}}{\partial p_{i}^{\ell}} & =\delta_{i j} .
\end{aligned}
$$

Putting this all together, an extreme entropy state must have

$$
\ln p_{i}^{\ell}=\ln \Pi_{\ell}-1-\beta\left\langle\Psi_{i}\right\rangle \sigma_{\ell}+\alpha \sigma_{\ell}+\lambda_{i},
$$

where a constant $\Delta x^{2}$ has been absorbed into $\alpha$ and $\beta$. Solving for $p_{i}^{\ell}$ yields the equilibrium distribution

$$
p_{i}^{\ell}=Z_{i}^{-1} e^{\left(-\beta\langle\Psi\rangle_{i}+\alpha\right) \sigma_{\ell}} \Pi_{\ell}
$$

where $\beta$ and $\alpha$ can be chosen to satisfy the constraints (27) and (28), and the partition function $Z_{i}$ is given by

$$
Z_{i}=\sum_{\ell} e^{\left(-\beta\langle\Psi\rangle_{i}+\alpha\right) \sigma_{\ell}} \Pi_{\ell}
$$

The relation (30) can be combined with (23) and (24) to solve for prospective mean fields. The mean stream function $\langle\Psi\rangle$ is found by solving

$$
\sum_{j} \Delta_{i j}\left\langle\Psi_{j}\right\rangle=\frac{\sum_{\ell} \sigma_{\ell} e^{\left(-\beta\left\langle\Psi_{i}\right\rangle+\alpha\right) \sigma_{\ell}} \Pi_{\ell}}{\sum_{\ell} e^{\left(-\beta\left\langle\Psi_{i}\right\rangle+\alpha\right) \sigma_{\ell}} \Pi_{\ell}}-h\left(\boldsymbol{x}_{i}\right),
$$

together with the constraints (27) and (28).

The EHT theory is microcanonical with respect to the energy and circulation, in the sense that the parameters $\beta$ and $\alpha$ are chosen as Lagrange multipliers to ensure that the resulting mean field assumes desired values of these quantities. It is canonical with respect to higher order Casimir's in the sense that the fine-scale vorticity is specified as a distribution.

\section{Numerical verification of the HPM statistical equilibrium theories}

In this section, we compare the predicted mean fields $\langle\boldsymbol{q}\rangle$ and $\langle\boldsymbol{\Psi}\rangle$ of the discrete equilibrium statistical models from the previous sections, with long time average mean fields $\overline{\mathbf{q}}$ and $\overline{\boldsymbol{\Psi}}$ computed from numerical simulations with the HPM method, under the assumption that the simulated solution is approximately ergodic. It should be noted that the probability distributions (21) and (30) predict much more than just the mean states $\langle\boldsymbol{q}\rangle$ and $\langle\boldsymbol{\Psi}\rangle$, so our comparison is necessarily a limited one. Yet from a numerical point of view, correct representation of the mean state is a minimal requirement, as it sets the statistical background for dynamics.

The theoretical mean fields (20) and (32) based on the Lagrangian and Eulerian statistical models are computed numerically. Due to point-wise conservation of PV on the particles, and the construction (11), the space of grid-based PV fields is bounded, as is the partition function (31). For a given particle field $\boldsymbol{Q}$ and values for the constraints we solve for the mean fields (20) and (32) plus associated Lagrange multipliers using a modified Newton iteration. These fields are compared with average fields generated by long-time simulations.

For the numerical simulations we use the test setup of Abramov and Majda [1]. We choose grid resolution $M=24$. The topography is a function of $x$ only, specifically

$$
h(x, y)=0.2 \cos x+0.4 \cos 2 x,
$$

which is intended to make departures from Gaussian PV theory readily observable (see below).

The integrations were carried out using a step size of $\Delta t=2 / M$ on the interval $t \in\left[0, t_{0}+T\right]$. The solutions are averaged over the time interval $t \in\left[t_{0}, t_{0}+T\right]$, where $t_{0}$ is the time required for decorrelation of the initial condition. In all experiments we use $t_{0}=10^{3}$ and $T=10^{4}$. Longer simulations with $T=10^{6}$ were also run with no observable difference in the results. The implicit midpoint rule nonlinear relations were solved to machine precision.

All simulations were carried out with $\kappa=1$. Together with the low value of $M$, this implies the simulations were highly under-resolved. This has the double effect of allowing us to stretch the limits of the discrete statistical models, for which 
various approximations were made, and to allow the system to sample the available phase space (assuming ergodicity) in a reasonably short simulation interval.

We construct initial conditions with a desired prior distribution and energy value. The mean state (30) is fully defined by these quantities. If the dynamics is sufficiently ergodic, then the time average mean stream function $\overline{\boldsymbol{\Psi}}$ and mean potential vorticity $\overline{\boldsymbol{q}}$ should agree with the ensemble averages (24) and (23).

Given a continuous prior distribution on vorticity $\Pi(\sigma)$, we define particle PV values $Q_{k}$ as follows. The number of particles is $K=\kappa^{2} M^{2}$. We discretize the range of vorticity $\sigma$ into $L$ equal partitions of size $\Delta \sigma$ where

$$
\sigma_{\ell}=\sigma_{0}+\ell \Delta \sigma, \quad \ell=1, \ldots, L .
$$

We choose the number of particles with vorticity $\sigma_{\ell+1 / 2}=\left(\sigma_{\ell}+\sigma_{\ell+1}\right) / 2$ to be
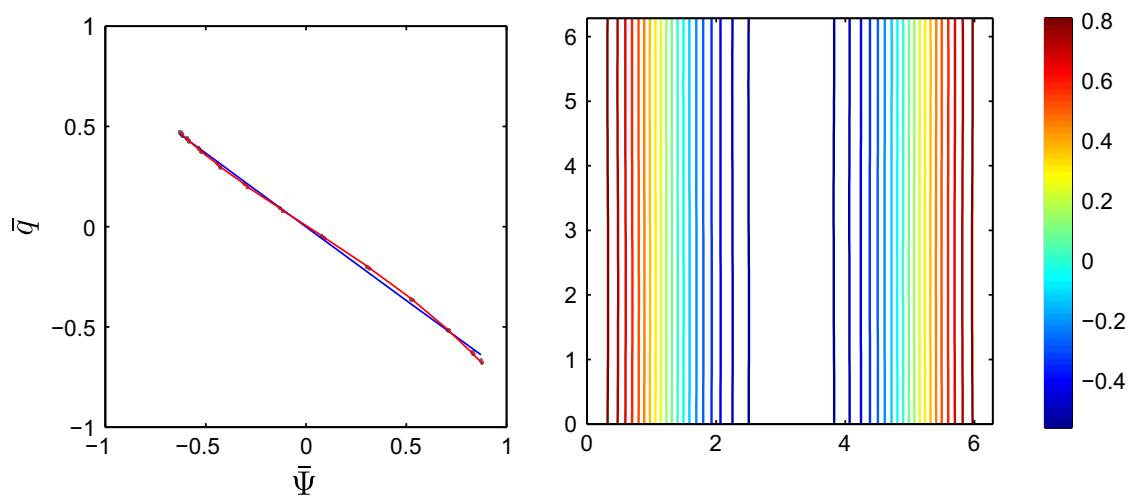

Fig. 3. Normally distributed PV on the particles. The scatter plot of mean fields (left) with linear fit. Mean stream function (right).
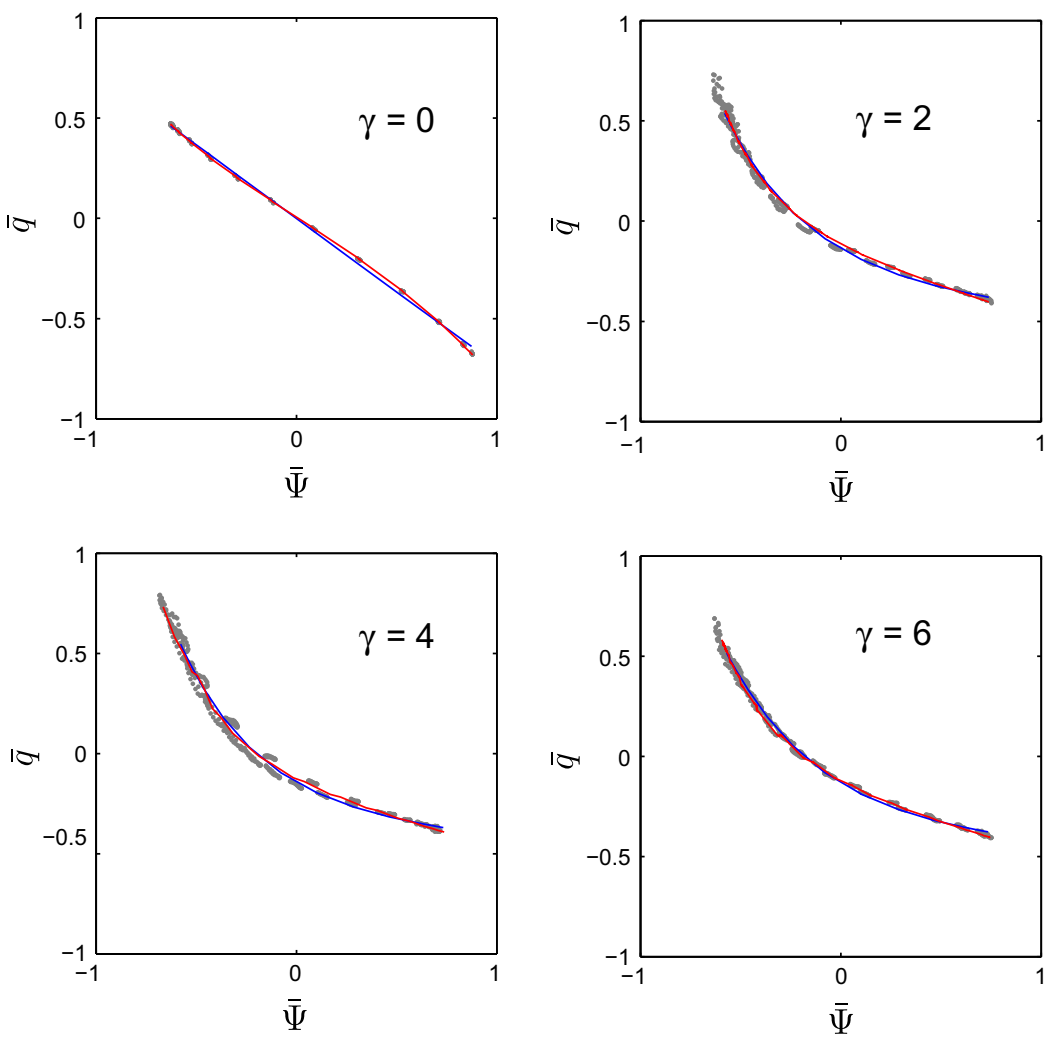

Fig. 4. Locus $\left(\bar{q}_{i}, \bar{\Psi}_{i}\right)$ for skewed PV distributions, $\gamma=0,2,4$, and 6 (grey points). The theoretical prediction based on (21) is shown in red and that based on (30) is shown in blue. (For interpretation of the references in color in this figure legend, the reader is referred to the web version of this article.) 


$$
K_{\ell+1 / 2}=\left\lfloor K \int_{\sigma_{\ell}}^{\sigma_{\ell+1}} \Pi(\sigma) d \sigma\right\rfloor .
$$

Any remainder particles are assigned the values of the consecutive most probable level sets.

The particles are initially placed on a uniform grid of spacing $\Delta a=\Delta x / \kappa$ in each direction. Using Monte Carlo simulations, the PV values are randomly permuted until a configuration is found within desired total energy (grid function) $H_{0} \pm 0.01$. In all simulations, the target energy was $H_{0}=7$, and the total circulation was $C_{1}(\boldsymbol{Q})=0$, consistent with [1]. The Lagrange multipliers $\beta$ and $\alpha$ follow from the constraints of total energy and circulation.

\subsection{Normally distributed PV}

From the classical energy-enstrophy theory of Kraichnan and others $[15,27,4]$ it is known that if the PV field is normally distributed, the mean field relation should be linear of the form (7). To verify this for the HPM method, we draw the particle vorticities from a zero-mean Gaussian prior distribution

$$
Q_{k} \sim \Pi(\sigma)=\exp \left(-\frac{\sigma^{2}}{2 \theta^{2}}\right) .
$$

In this case the EHT theory yields (in the semi-discrete case)

$$
p_{i}(\sigma)=Z_{i}^{-1} \exp \left(-\beta\left\langle\Psi_{i}\right\rangle \sigma\right) \Pi(\sigma),
$$

which is continuous in the PV $\sigma$. This density can be exactly integrated to yield the linear mean field relation

$$
\left\langle q_{i}\right\rangle=-\beta \theta^{2}\left\langle\Psi_{i}\right\rangle
$$

We choose $\beta$ and $\theta$ to specify energy $H_{0}=7$ and enstrophy $C_{2}=40$.

In the left panel of Fig. 3, the locus of data points $\left(\bar{\Psi}_{i}, \bar{q}_{i}\right)$ is plotted for the time-averaged fields. The vorticity-stream function relation is nearly linear as predicted. Due to the finite sampling of the Gaussian distribution, the simulation data is not precisely linear. The Eulerian statistical model (30) yields a more linear mean field prediction, but the Lagrangian statistical model (21) more precisely fits the simulation data.

$$
\gamma=0
$$

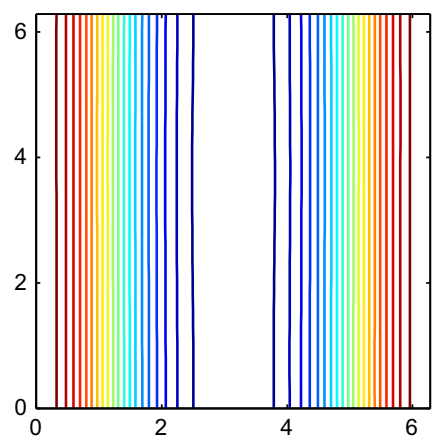

$$
\gamma=4
$$

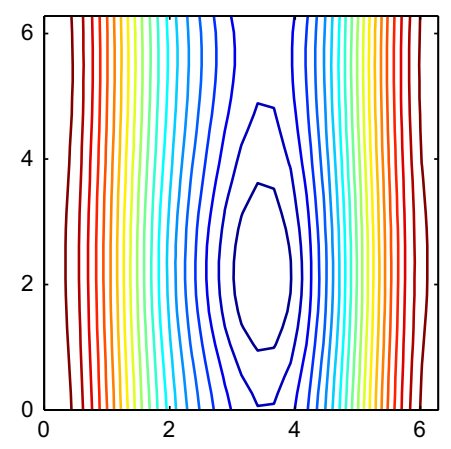

$\gamma=2$

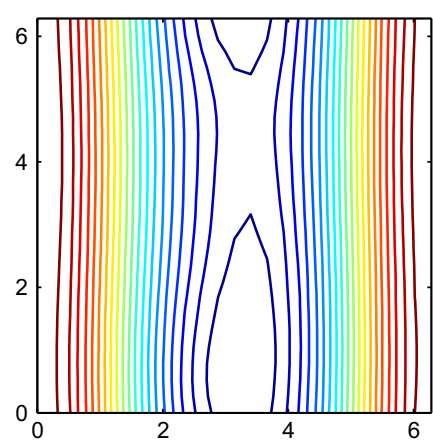

$\gamma=6$

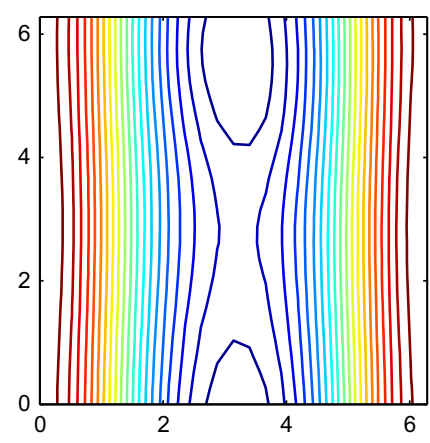

0.6
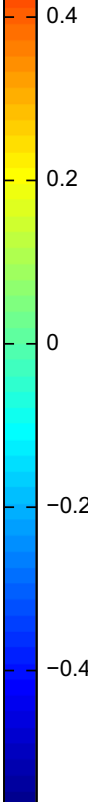

Fig. 5. Mean stream functions corresponding to Fig. 4. For nonzero skewness $\gamma \neq 0$ the stream function is two-dimensional, despite one-dimensional topography. 
Due to the linearity and isotropy of (7) and (8), the mean stream function $\langle\psi\rangle$ satisfies a Helmholtz equation and is expected to be independent of $y$ due to the special choice of topography. In the right panel we observe that the mean stream function is indeed independent of $y$.

\subsubsection{Skew PV distributions}

In [1], Abramov and Majda show that nonzero values of the third moment of potential vorticity can cause significant deviation from the statistical predictions of the normally distributed PV case. They use the Poisson discretization of Zeitlin [29] to solve the QG model. On an $M \times M$ grid the Zeitlin method conserves energy and approximations of the first $M$ moments of potential vorticity $\widehat{C}_{r}, r=1, \ldots, M$.

We generate initial conditions $\boldsymbol{Q}$ from the shifted gamma-distribution [5]:

$$
\Pi(\sigma)=\frac{1}{C_{2}|\lambda|} R\left(\frac{1}{C_{2} \lambda}\left(\sigma+\lambda^{-1}\right) ; \frac{1}{C_{2} \lambda^{2}}\right),
$$

where $R(z ; a)=\Gamma(a)^{-1} z^{a-1} e^{-z}$ for $z \geqslant 0$ and $R=0$ otherwise, and

$$
\gamma=\frac{C_{3}}{C_{2}^{3 / 2}}=2 C_{2}^{1 / 2} \lambda
$$

is the skewness of the distribution. We take $C_{2}=40$ and $\gamma=0,2,4$, and 6 to compare the results of [1] with the HPM method.

Fig. 4 gives the $\left(\bar{\Psi}_{i}, \bar{q}_{i}\right)$ loci for the time-averaged fields, for these values of $\gamma$. Fig. 5 illustrates the associated mean stream functions. The solutions are reminiscent of those reported in [1], but there are some differences due to the details of the methods.

For the case $\gamma=0$, the energy-enstrophy theory predicts a linear relation (7) between mean PV and mean stream function, as well as a layered mean stream function. These predictions are confirmed in the upper left panels of Figs. 4 and 5 . For $\gamma>0$, there is significant nonlinearity in the mean field relation and vortical structures observable in the mean stream function.
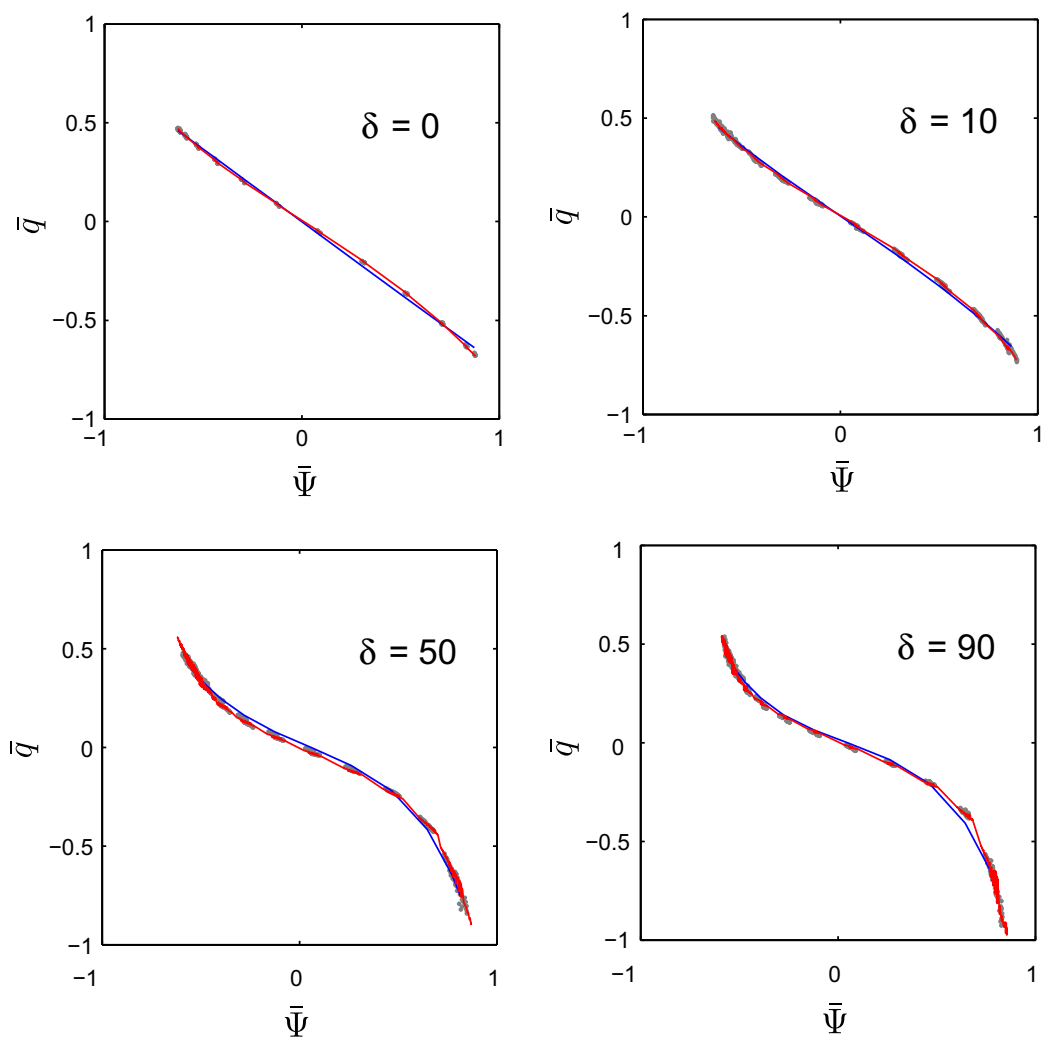

Fig. 6. Locus $\left(\bar{q}_{i}, \bar{\Psi}_{i}\right)$ for kurtotic PV distributions, $\delta=0,10,50$, and 90 (grey points). The theoretical prediction based on (21) is shown in red and that based on (30) is shown in blue. (For interpretation of the references in color in this figure legend, the reader is referred to the web version of this article.) 
$\delta=0$

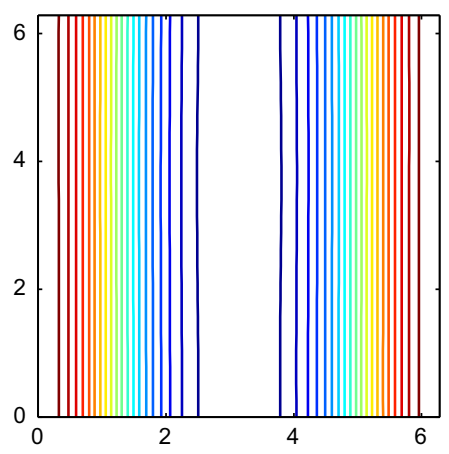

$\delta=50$

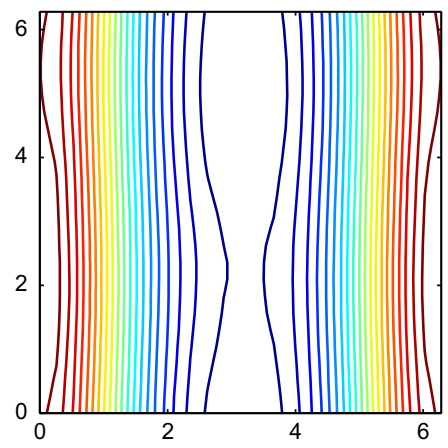

$\delta=10$

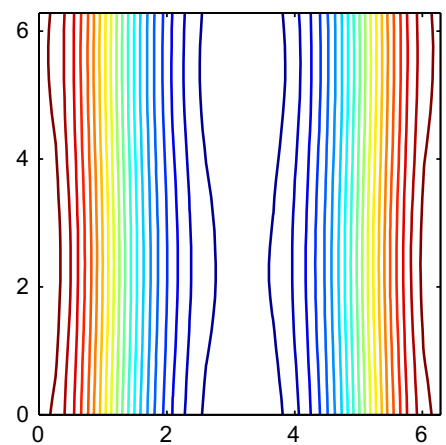

$\delta=90$

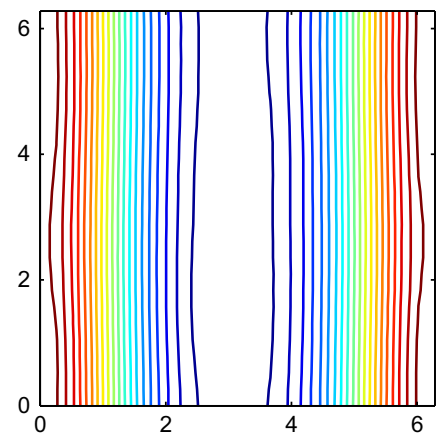

0.6

$-0.4$

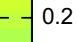

$-0$

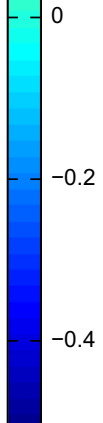

Fig. 7. Mean stream functions corresponding to Fig. 6. For nonzero skewness $\delta \neq 0$ the stream function is two-dimensional, despite one-dimensional topography.

Also shown in Fig. 4 are the theoretical mean states predicted by the discrete statistical equilibrium theories in Sections 4 and 5. The Lagrangian statistical model (20) is shown in red and the Eulerian model (32) in blue. Both models predict the mean states very well.

\subsubsection{PV distributions with kurtosis}

Abramov and Majda [1] also conjecture that the higher-order moments $C_{r}, r \geqslant 4$, are statistically irrelevant for predicting the large-scale mean flow, based on the observation that the experiments agreed well with the energy-enstrophy mean field theory (7) in the case $\gamma=0$, despite the fact that the moments $\widehat{C}_{r}, r \geqslant 4$ were nonzero as arbitrarily determined by their initialization procedure, and conserved by the method.

To investigate this conjecture we choose initial distributions $\boldsymbol{Q}$ having skewness $\gamma=0$ and nonzero kurtosis (scaled fourth moment of PV),

$$
\delta=\frac{C_{4}}{C_{2}^{2}}-3 .
$$

In this case we generated the initial particle PV field by first drawing the $Q_{k}$ from a uniform distribution and then projecting onto the constraint set $\left\{H_{0}=7, C_{1}=0, C_{2}=40, C_{3}=0, C_{4}=(\delta+3) C_{2}^{2}\right\}$.

Fig. 6 shows the mean field relations $\left(\bar{q}_{i}, \bar{\Psi}_{i}\right)$ for increasing $\delta=0,10,50$, and 90 . The corresponding mean stream functions are shown in Fig. 7. We observe that nontrivial kurtosis may also significantly influence the mean field statistics, which disproves the conjecture of [1].

Again we observe in Fig. 6 that both (20) and (32) do an excellent job of predicting the mean states.

\section{Conclusions}

The HPM method, as adapted for 2D incompressible flow, conserves total energy by construction. Each particle is assigned a constant value of potential vorticity at initialization, and this discrete PV field is conserved point-wise, as the particles evolve in the divergence-free flow. In this sense, PV conservation induces no reduction in degrees of freedom on the dynamics. At the coarse scale, the vorticity field on the mesh satisfies conservation of energy and total circulation, but exhibits sig- 
nificant drift for nonlinear PV functionals. This is consistent with what would be observed if a coarse-graining procedure were applied to a real inviscid flow.

A maximum entropy theory based only on energy and circulation would predict zero mean flow (i.e. $\langle\psi\rangle \equiv 0$ ). In contrast to this prediction, we have demonstrated in this paper that the HPM method has a much richer statistical mechanics, with nonlinear mean field relations similar to those of [1], and consistent with the canonical EHT theory [10]. In particular, we have demonstrated that both the third and fourth moments of PV $\left(\mathcal{C}_{3}\right.$ and $\left.\mathcal{C}_{4}\right)$ can significantly affect the mean field relation. The latter result disproves a conjecture of Abramov and Majda [1].

We have also presented two statistical mechanics models for the HPM method, a Lagrangian and an Eulerian model. The Eulerian model is analogous to the EHT theory, which uses a canonical treatment of fine-scale vorticity in the form of a prior distribution, and enforces conservation of energy and total circulation through the use of Lagrange multipliers. In the present case, the prior distribution characterizes the particle vorticity field, and the energy and circulation are conserved at the grid scale. The Lagrangian statistical model is constructed on the phase space of particle positions, considering each particle to be immersed in a reservoir defined by the mean flow. The fine scale, particle statistics are given by canonical ensemble distributions, and the temperature parameter is used as a Lagrange multiplier to enforce energy conservation. Mean states computed with both statistical models compare very well with the long-time simulation data.

Although PV is simply assigned to particles and its conservation does not imply any dynamic constraint on the evolution, an appeal to the Lagrangian statistical model suggests that a particle's PV value determines its response to the mean flow, and thereby its residence time in any particular region of the flow domain. Via the basis functions $\phi$, the local residence time is translated to the grid scale where the coarse-grain dynamics is governed by energy conservation.

The essential ingredients of the Miller/Robert and EHT statistical theories are the fine scale point-wise advection of PV and the coarse scaling associated with the stream function, under the constraint of energy conservation. The HPM method retains these features under discretization, and for this reason its equilibrium statistics are analogous to those theories. From the numerical experiments we can conclude that the HPM method is free of artificial dissipation or other errors that might destroy the equilibrium statistical mechanics. For the experiments conducted, the discrete dynamics is also sufficiently ergodic that the averages are well approximated.

\section{Acknowledgments}

We wish to acknowledge helpful discussions with Oliver Bühler, Jacques Vanneste and Sebastian Reich.

\section{References}

[1] R. Abramov, A.J. Majda, Statistically relevant conserved quantities for truncated quasi-geostrophic flow, Proc. Natl. Acad. Sci. USA 100 (7) (2003) 38413846.

[2] O. Bokhove, M. Oliver, Parcel Eulerian-Lagrangian fluid dynamics of rotating geophysical flows, Proc. R. Soc. Lond. Ser. A: Math. Phys. Eng. Sci. 462 (2073) (2006) 2575-2592.

[3] O. Buhler, Statistical mechanics of strong and weak point vortices in a cylinder, Phys. Fluid 14 (2002) $2139-2149$.

[4] G. Carnevale, J. Frederiksen, Nonlinear stability and statistical mechanics of flow over topography, J. Fluid Mech. 175 (1987) 157-181.

[5] P.-H. Chavanis, Statistical mechanics of 2D turbulence with a prior vorticity distribution, Physica D 237 (14-17) (2008) 1998-2002.

[6] C. Cotter, S. Reich, Geometric integration of a wave-vortex model, Appl. Numer. Math. 48 (2004) 293-305.

[7] C.J. Cotter, J. Frank, S. Reich, Hamiltonian particle-mesh method for two-layer shallow-water equations subject to the rigid-lid approximation, SIAM J. Appl. Dyn. Syst. 3 (1) (2004) 69-83 (electronic).

[8] G.-H. Cottet, P.D. Koumoutsakos, Vortex Methods: Theory and Practice, Cambridge University Press, Cambridge, 2000.

[9] S. Dubinkina, J. Frank, Statistical mechanics of Arakawa's discretizations, J. Comput. Phys. 227 (2007) 1286-1305.

[10] R.S. Ellis, K. Haven, B. Turkington, Nonequivalent statistical equilibrium ensembles and refined stability theorems for most probable flows, Nonlinearity 15 (2) (2002) 239-255.

[11] J. Frank, G. Gottwald, S. Reich, A Hamiltonian particle-mesh method for the rotating shallow-water equations, Meshfree Methods for Partial Differential Equations (Bonn, 2001), Lect. Notes Comput. Sci. Eng., vol. 26, Springer, Berlin, 2003. pp. 131-142.

[12] J. Frank, S. Reich, Conservation properties of smoothed particle hydrodynamics applied to the shallow water equation, BIT 43 (1) (2003) 41-55.

[13] J. Frank, S. Reich, The Hamiltonian particle-mesh method for the spherical shallow water equations, Atmos. Sci. Lett. 5 (2004) 89-95.

[14] E. Hairer, C. Lubich, G. Wanner, Geometric Numerical Integration, Springer Series in Computational Mathematics, second ed., vol. 31, Springer-Verlag, Berlin, 2006 (structure-preserving algorithms for ordinary differential equations).

[15] R. Kraichnan, Statistical dynamics of two-dimensional flow, J. Fluid Mech. 67 (1975) 155-175.

[16] B. Leimkuhler, S. Reich, Simulating Hamiltonian Dynamics, Cambridge Monographs on Applied and Computational Mathematics, vol. 14, Cambridge University Press, Cambridge, 2004

[17] D. Lynden-Bell, Statistical mechanics of violent relaxation in stellar systems, Mon. Not. R. Astr. Soc. 136 (1967) 101-121.

[18] A.J. Majda, X. Wang, Non-linear Dynamics and Statistical Theories for Basic Geophysical Flows, Cambridge University Press, Cambridge, 2006.

[19] R.I. McLachlan, Explicit Lie-Poisson integration and the Euler equations, Phys. Rev. Lett. 71 (19) (1993) 3043-3046.

[20] J. Miller, Statistical mechanics of Euler equations in two dimensions, Phys. Rev. Lett. 65 (17) (1991) 2137-2140.

[21] J. Miller, P.B. Weichman, M.C. Cross, Statistical mechanics, Euler's equation, and Jupiter's Red Spot, Phys. Rev. A 45 (4) (1992) $2328-2359$.

[22] L. Onsager, Statistical hydrodynamics, Nuovo Cimento 6 (Suppl. 2) (1949) 279-287.

[23] J. Pedlosky, Geophysical Fluid Dynamics, second ed., Springer, New York, 1987.

[24] R. Robert, A maximum-entropy principle for two-dimensional perfect fluid dynamics, J. Statist. Phys. 65 (3-4) (1991) $531-553$.

[25] R. Robert, J. Sommeria, Statistical equilibrium states for two-dimensional flows, J. Fluid Mech. 229 (1991) $291-310$.

[26] R. Salmon, Lectures on Geophysical Fluid Dynamics, Oxford University Press, New York, 1998.

[27] R. Salmon, G. Holloway, M. Hendershott, The equilibrium statistical mechanics of simple quasi-geostrophic models, J. Fluid Mech. 75 (1976) 691-703.

[28] S. Shin, S. Reich, Hamiltonian particle-mesh simulations for a non-hydrostatic vertical slice model, Atmos. Sci. Lett. 10 (2009) $233-240$.

[29] V. Zeitlin, Finite-mode analogues of 2D ideal hydrodynamics: coadjoint orbits and local canonical structure, Physica D 49 (3) (1991) 353-362. 